\title{
Trial Summary Domain
}

National Cancer Institute

\section{Source}

National Cancer Institute. Trial Summary Domain. NCI Thesaurus. Code C53483.

A subject domain utilized for the submission of information encompassing and representing data, vocabulary or records related to trial summary. 